\title{
Improvement in hearing functions after successful carotid body tumor resection
}

\author{
Yasin Özden (D), Ahmet Bolukçu (D) \\ Department of Cardiovascular Surgery, University of Health Sciences, Dr. Siyami Ersek Thoracic and Cardiovascular Surgery Training and Research Hospital, Istanbul, Turkey
}

Received: September 29, 2021 Accepted: October 26, 2021 Published online: November 12, 2021

\begin{abstract}
Carotid body tumors (CBTs), also known as paragangliomas, are rare neuroendocrine hypervascularized neoplasms. They are slowly growing pulsatile masses, usually benign, near the carotid arteries. Besides the endocrinological effects of the tumor, compression and hypervascular invasion effects can be also seen. A 63-year-old female patient who had hearing loss in the right ear was admitted to our clinic. Coil embolization was performed to the major arteries feeding the tumor before total excision of the tumor. A decrease in the hearing loss was observed after the operation which might be due to compression and hypervascular steal effects of the tumor. In conclusion, hearing functions can be improved with success CBT resection.
\end{abstract}

Keywords: Carotid artery, carotid body tumor, glomus tumor, hearing loss paraganglioma.

Carotid body tumors (CBTs), also known as paragangliomas, are rare neuroendocrine hypervascularized neoplasms that arise on the glomus derived from the embryonic neural crest within the carotid bifurcation. The incidence is 1 to $2 / 100,000$, and they have usually a benign character. Less than $10 \%$ of the tumors are malignant. ${ }^{[1]}$

Carotid body tumors are painless, slowly growing pulsatile masses, usually lateral to the hyoid bone. Symptoms may vary depending on the site of the invasion and the compression. Tinnitus and hearing loss can be seen, particularly in paragangliomas with tympanic and jugular extension. ${ }^{[2]}$

In this report, we present a case with a CBT that caused hearing loss for two years whose symptoms relieved after surgical excision of the tumor following preoperative coil embolization.

\section{CASE REPORT}

A 63-year-old female patient who had no chronic disease other than hypertension and hearing loss in the right ear was admitted to our clinic suffering from swelling in the right neck region. The physical examination revealed a palpable pulsatile mass in the neck extending laterally and superiorly from the bifurcation level of the common carotid artery. On magnetic resonance imaging, we detected a hypervascular hyperintense mass approximately
$55 \times 45 \mathrm{~mm}$ in size with smooth borders causing displacement and extrinsic compression to the parotid gland. No abnormalities were found in the patient's catecholamine and other laboratory tests. On carotid angiography, major feeding branches of the tumor were visualized (Video 1,2). The branches close to the bifurcation arising from the temporal artery were embolized to decrease bleeding during surgical excision of the tumor and it was confirmed that the tumor blood supply was minimized by re-imaging. The patient underwent surgery for $36 \mathrm{~h}$ after embolization. During the operation, we observed that the tumor arose from the carotid bifurcation and covered the proximal internal carotid artery, external carotid artery, and the distal common carotid artery completely with the extensions to the mastoid bone toward the skull base, mandibular region, and the thyroid gland (Video 3). The tumor was evaluated as a Shamblin type 3 tumor. It was totally excised using the bipolar cautery and scissors (Video 4). A total of $110 \mathrm{~mL}$ bleeding was detected during the operation.

Corresponding author: Yasin Özden, MD. SBÜ Dr. Siyami Ersek Göğüs Kalp ve Damar Cerrahisi Eğitim ve Araştırma Hastanesi, Kalp ve Damar Cerrahisi Kliniği, 34668 Üsküdar, İstanbul, Turkey.

Tel: +90 216 - 5424444 e-mail: dr.yasinozden@gmail.com

\section{Citation:}

Özden Y, Bolukçu A. Improvement in hearing functions after successful carotid body tumor resection. Cardiovasc Surg Int 2021;8(3):162-165. 


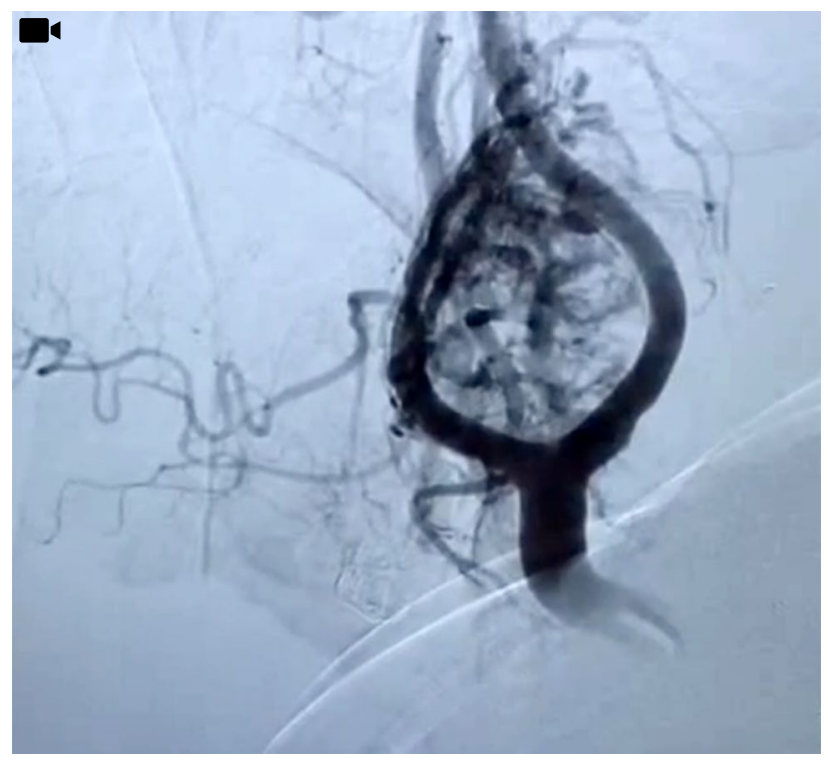

Video 1. Location of the tumor relative to the carotid arteries in angiographic imaging.

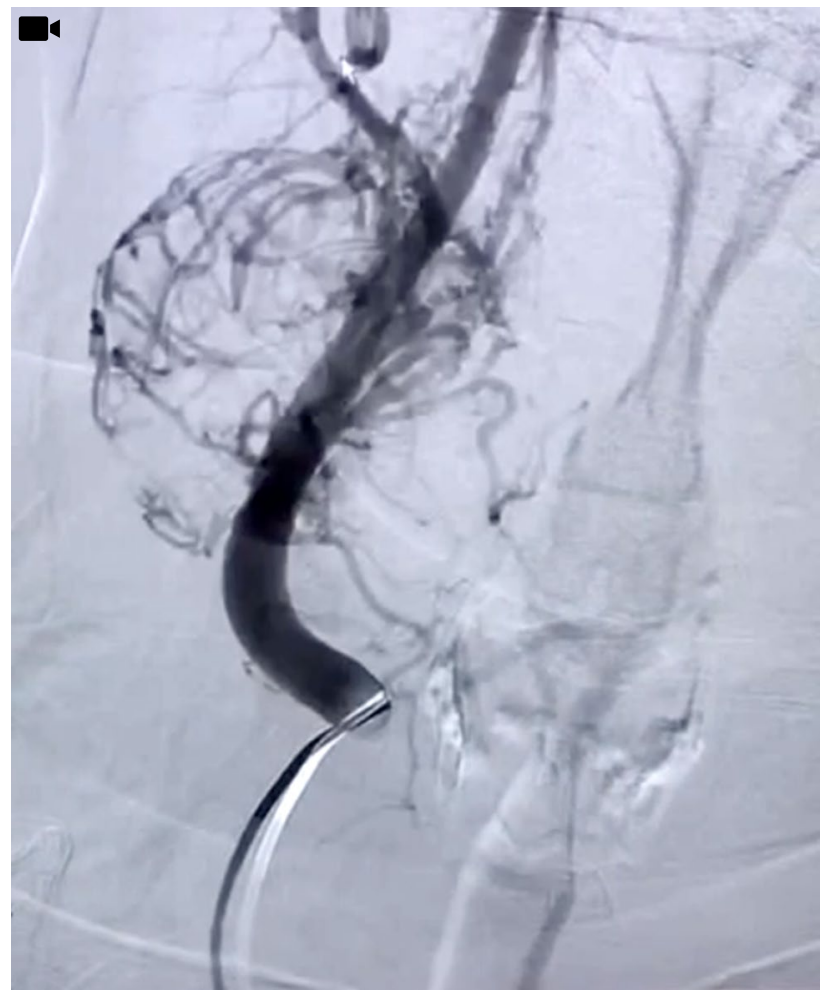

Video 2. Angiographic imaging of the vessels feeding the tumor.

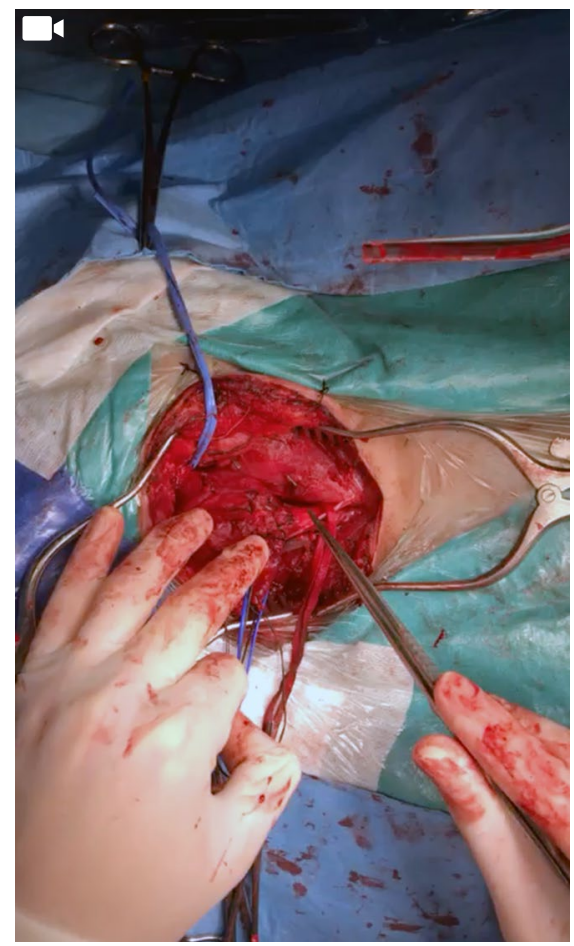

Video 3. The relation of the tumor with other tissues in surgical exposure.

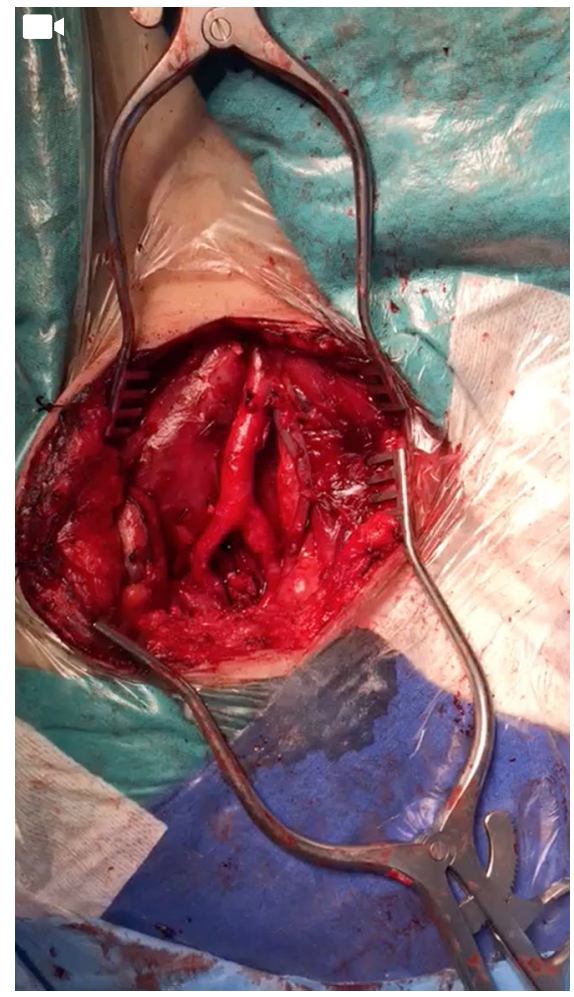

Video 4. Tumor totally excised carotid arteries. 
No neurological deficit was seen after surgery. The patient who used a hearing aid due to severe hearing loss in the right ear for two years before the operation and could not hear normal speech sounds in his right ear without the device reported that she could hear rough sounds without the hearing aid after the operation. Compared to the previous hearing test, the patient's hearing loss decreased clinically and $15 \mathrm{~dB}$ in the audiogram. The patient was discharged on Day 4 after the operation. The pathology report revealed a unifocal paraganglioma with solid and alveolar growth patterns; without mitosis, composite tumor elimination, transcapsular and lymphovascular invasion. The specimen had a diffuse strong staining with synaptophysin and the surgical margins were negative. A written informed consent was obtained from the patient.

\section{DISCUSSION}

Conventional surgery, endovascular embolization and rarely radiotherapy can be used in the treatment of CBTs. Endovascular embolization before classical surgery is controversial. Hypervascularization of the tumor is associated with an increased risk of bleeding during the operation and increased blood transfusion. To prevent this, endovascular embolization prior to surgery may reduce bleeding by closing the major vessels feeding the tumor. However, the risk of cerebrovascular events and vascular rupture increases with this procedure. In a study of 29 patients with CBTs conducted by Zhang et al., ${ }^{[3]}$ significantly less bleeding during the operation and shorter operative times were observed in the patients who underwent preoperative endovascular embolization; however, no relationship was found with the nerve damage. In our case, we performed an endovascular embolization before surgery and we had a total bleeding of $110 \mathrm{~mL}$ during the surgical dissection of the tumor with more than one feeding artery. We did not encounter any cerebrovascular diseases after endovascular or surgical procedures. Hearing loss due to $\mathrm{CBT}$ is not common in the literature. There is a limited number of studies and case reports related to this. Temmel et al. ${ }^{[4]}$ presented a case with decreased neurosensory hearing loss after CBT excision, but they could not reveal a clear pathophysiological condition to explain this situation. However, they focused on two possible considerations. First, the mass with neurosecretory features could affect the sympathetic ganglia via neurotransmitters. The second and more effective, the sympathetic ganglia were affected by the compression effect of the tumor. In a study, Lord and Chambers ${ }^{[5]}$ reported that familial genetically inherited (autosomal dominant) CBTs with hearing loss were transferred to the lower generations by genetic inheritance and the source of hearing loss was more frequently accompanied by genetically inherited CBT. In our case, we did not consider genetic inheritance, as there was no CBT or any other relatives with hearing loss in her family. As a result of total surgical excision, we removed the pressure on neighboring tissues and increased the perfusion to other tissues and organs in this area, as the excessive blood flow to the tumor in the neck area was removed.

In conclusion, we operate patients with CBT in our clinic. However, hearing loss is extremely rare in these patients. In general, the main complaints are pulsatile masses in the neck and tinnitus. This case is the first in our clinic presenting with CBT accompanied by hearing loss. We believe that the mass has the effect of compression on the sympathetic ganglia in this region rather than the neuroendocrine effect of the tumor, resulting in both a decrease in neural conduction pathways and vasoconstriction in the vertebrobasilar system. Another possible explanation is that the hypervascular tumor has a steal effect on cochlear perfusion. If we consider that there is a mass (particularly located superiorly) and steal effect in non-familial CBT cases, hearing loss may be improved in these patients after surgical operation. However, further studies are needed to establish a definite conclusion on this subject.

\section{Declaration of conflicting interests}

The authors declared no conflicts of interest with respect to the authorship and/or publication of this article.

\section{Funding}

The authors received no financial support for the research and/or authorship of this article.

\section{REFERENCES}

1. Hoang VT, Trinh CT, Lai TAK, Doan DT, Tran TTT. Carotid body tumor: A case report and literature review. J Radiol Case Rep 2019;13:19-30.

2. Woolen S, Gemmete JJ. Paragangliomas of the head and neck. Neuroimaging Clin N Am 2016;26:259-78.

3. Zhang J, Fan X, Zhen Y, Chen J, Zheng X, Ma B, et al. Impact of preoperative transarterial embolization of carotid body tumor: A single center retrospective cohort experience. Int J Surg 2018;54:48-52. 
4. Temmel AF, Kierner AC, Muhm M, Gstoettner W. Reversible sensorineural hearing impairment induced by a carotid body tumor. Eur Arch Otorhinolaryngol 1999;256:466-9.
5. Lord RS, Chambers AJ. Familial carotid body paragangliomas and sensorineural hearing-loss: A new syndrome. Cardiovasc Surg 1999;7:134-8. 